\title{
Collapse of the Nonlinear Spin Dipole Wave Pulses of the Millimeter Wave Range in the YIG Films
}

\author{
V. Grimalsky ${ }^{a, *}$, S. Koshevaya ${ }^{a}$ And O. Kolokoltsev ${ }^{b}$ \\ ${ }^{a}$ CIICAp, Autonomous University of State Morelos (UAEM), Cuernavaca, ZP 62209, Mor., Mexico \\ ${ }^{b}$ CCADET, Autonomous National University of Mexico (UNAM), Mexico, ZP 04510, D.F., Mexico
}

\begin{abstract}
The spatiotemporal collapse of the spin-dipole wave pulses of the millimeter wave range is investigated theoretically. The dispersion and the diffraction coefficients of spin-dipole wave have been calculated, when the retardation has been taken into account. It is demonstrated that YIG films are suitable for observing the wave collapse in the millimeter wave range, due to low dissipation and quite high values of dispersion and diffraction coefficients. In the hexaferrite films, despite the higher values of the dispersion and the diffraction coefficients, the dissipation prevents the formation of the spatiotemporal collapse. The additional focusing of the pulse can increase the nonlinear pulse compression. The numerical simulations have confirmed this result.
\end{abstract}

PACS: 85.70.Ge, 75.30.Ds, 84.40.-x, 42.65.Tg, 05.45.Yv

\section{Introduction}

The nonlinear wave propagation and the interaction in the solids are of the great interest during many years. The big attention has been given to the solitons and the related phenomena like the self-focusing and the wave collapse. The variety of the solitons was presented in the optics [1]. In the microwave and millimeter wave ranges the nonlinear waves have been investigated in the semiconductor electron plasmas [2] and in the ferrite films [3-11]. In the present paper the waves in ferrite films are considered, because these films are characterized by the high nonlinearity and the low losses.

Most of the interesting results are devoted to the propagation of the nonlinear waves in the ferrite films along the bias magnetic field, for example, the backward volume magnetostatic waves (BVMSW) [4-10], where both solitons and collapsing nonlinear wave pulses have been observed [3-5]. The detailed theoretical and experimental investigations were performed in the centimeter wave range, at the frequencies $f \approx 3-20 \mathrm{GHz}$, with YIG films. It is of the interest to investigate the nonlinear spin-dipole wave propagation in the millimeter wave range, $f \approx 30-60 \mathrm{GHz}$. At those frequencies, the hexaferrite films can be suitable, due to the higher magnetization and the essential magnetic anisotropy field [3]. But the hexaferrite films possess the strong dissipation compared with the YIG films. It seems important to consider the nonlinear wave propagation in the YIG films under the higher bias magnetic fields $H_{0} \geq 10 \mathrm{kOe}$ that are accessible for the experiments, because the dissipation is a lim-

* corresponding author; e-mail: v_grim@yahoo.com iting factor for the observing of the solitons and the wave collapse. In this paper the absolute (Gaussian) units are used.

The propagation of the linear and the nonlinear spin-dipole wave (SDW) in the centimeter wave range is usually considered in a quasistationary approximation, where the electromagnetic (EM) retardation is neglected, so-called the magnetostatic waves. In the millimeter wave range the applicability of the quasistationary approximation is doubtful, because the longitudinal wave number of SDW can be comparable with one of the electromagnetic wave $(\omega / c) \varepsilon_{f}^{1 / 2} \approx 40 \mathrm{~cm}^{-1}$. For a generation of the bright temporal solitons and the spatial ones it is necessary to obtain the certain signs of the dispersion and the diffraction coefficients, respectively, if the sign of the nonlinearity coefficient is determined [3-6]. At the lower values of the wave number the hybridization of SDW with the EM one takes place. Even a small retardation may lead to an essential change of the wave dispersion because of the opposite directions of the group velocities of the BVMSW and the EM waves.

In the paper the propagation of the moderately nonlinear SDW in the YIG films and in the hexaferrite ones in the millimeter wave range is analyzed. The propagation is along the bias magnetic field. The dispersion and diffraction coefficients and the wave dissipation have been calculated both for the YIG films and for the hexaferrite ones. The values of the longitudinal wave numbers are specified where the propagation of the bright temporal solitons and the realization of the wave collapse are possible. The numerical simulations of the nonlinear wave propagation have been provided. The splitting with respect to the physical factors has been applied. A possibility of the spatial-temporal wave collapse of the SDW 
in the YIG films has been demonstrated. In the hexaferrite films the high dissipation prevents a realization of the wave collapse. The additional focusing of the SDW pulse can improve the nonlinear compression of the pulse.

\section{Dispersion and diffraction coefficients of the SDW in the millimeter wave range}

The propagation of the exchangeless SDW in the thin ferrite film along the bias magnetic field is considered. In the millimeter wave range, it is possible to use either hexaferrite films of the great magnetic anisotropy or to apply the high bias magnetic field $H_{0} \geq 10 \mathrm{kOe}$ in the case of the YIG films. The geometry of the problem is given in Fig. 1.

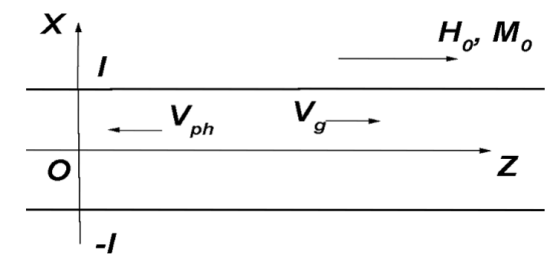

Fig. 1. Geometry of the problem.

The ferrite film $(|x|<l)$ is placed on a nonmagnetic substrate $(x<-l)$. The air is at $x>l$. Let us note that these SDW possess the opposite signs of the phase and group velocities. The frequency range of the propagation of the backward volume SDW is $\omega_{H}<\omega<\omega_{3} \equiv$ $\left[\omega_{H}\left(\omega_{H}+\omega_{M}\right)\right]^{1 / 2}$, where $\omega_{H}=\gamma H_{0}, \omega_{M}=4 \pi \gamma M_{0} ; H_{0}$ is the bias magnetic field jointly with the anisotropy field, $4 \pi M_{0}$ is the saturation magnetization, $\gamma$ is the gyromagnetic ratio. Usually the SDW propagation is considered at the frequencies $\omega \approx \omega_{3}$, and the following condition for the wave number $k$ is valid: $k l \ll 1$.

In the quasistationary approximation, it is possible to calculate analytically the dispersion coefficients for the BVMSW [3]. The following expansion of the frequency $\omega$ on the longitudinal $k_{z}$ and transverse $k_{y}$ wave numbers is used

$$
\begin{aligned}
& \Delta \omega \approx \frac{\partial \omega}{\partial k_{z}} \Delta k_{z}+\frac{1}{2} \frac{\partial^{2} \omega}{\partial k_{z}^{2}} \Delta k_{z}^{2}+\frac{\partial \omega}{\partial k_{y}^{2}} \Delta k_{y}^{2} \\
&+ \frac{\partial^{2} \omega}{\partial k_{z} \partial k_{y}} \Delta k_{z} \Delta k_{y}+\frac{\partial \omega}{\partial|A|^{2}}|A|^{2} \\
& \frac{\partial \omega}{\partial k_{z}} \approx-\frac{\omega_{M} l}{2}, \quad \frac{\partial^{2} \omega}{\partial k_{z}^{2}} \approx \omega_{M} l^{2}, \quad \frac{\partial \omega}{\partial|A|^{2}} \approx \frac{\omega_{M}}{4} \\
& \frac{\partial \omega}{\partial k_{y}^{2}} \approx-\frac{\omega_{M}\left(\frac{\omega_{M}}{\omega_{H}}+\frac{1}{2}\right) l^{2}}{2\left(1+\frac{\omega_{M}}{\omega_{H}}\right)^{1 / 2} \mu} \\
& \mu \approx \frac{\omega^{2}-\omega_{H}\left(\omega_{H}+\omega_{M}\right)}{\omega_{H} \omega_{M}} \\
& \text { dissipation: } \quad \omega^{\prime \prime} \approx\left(2 \frac{\omega_{H}}{\omega_{M}}+1\right) \frac{\omega_{M} \delta}{2} .
\end{aligned}
$$

Here $\Delta \omega=\omega-\omega_{0}, \Delta k_{z}=k_{z}-k_{z 0}, \Delta k_{y}=k_{y} ; \omega_{0}, k_{z 0}$ are the central values of the frequency and the wave number of the SDW packet, $k_{y 0}=0$. The parameter $\delta$ defines the dissipation in a ferrite material [10].

The numerically calculated dispersion relations for the linear SDW, the fundamental mode, are given in Fig. 2 both for the YIG films and the hexaferrite ones. The retardation has been taken into account in the parts (b), (c), (e), (f). The parameters for Fig. 2 are as follows: the thickness of the film is $2 l=10 \mu \mathrm{m}$ (parts (a), (b), (d), (e)), the bias magnetic field jointly with the anisotropy field is $H_{0}=12 \mathrm{kOe}$ (YIG) and $H_{0}=$ $10 \mathrm{kOe}$ for the hexaferrite; the saturation magnetization is 1760 Oe for the YIG and 5000 Oe for the hexaferrite. The dissipation parameter $\delta$ is $2 \times 10^{-4}$ for the YIG films and $10^{-3}$ for the hexaferrite ones; the last value is really even higher. One can see from Fig. 2, parts (c), (f) that the SDW possess the higher values of the dispersion in the thicker films, $2 l \geq 8 \mu \mathrm{m}$. The dielectric permittivities of the ferrite and the substrate are $\varepsilon_{f}=17$ and $\varepsilon_{d}=15$, respectively.

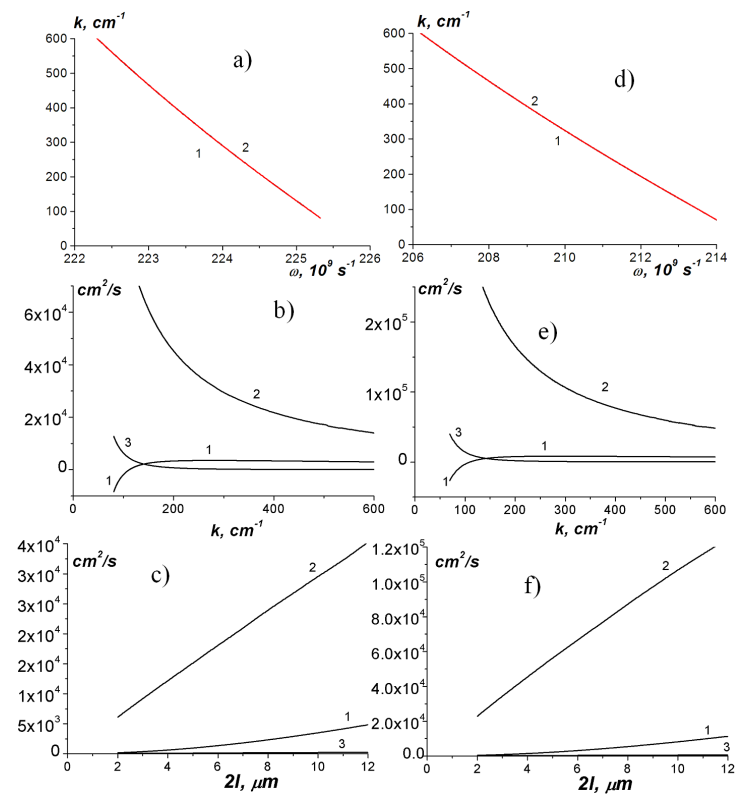

Fig. 2. Dispersion curves $k(\omega)$ and dispersion coefficients for the SDW in the YIG film $(a, b, c)$ and in the hexaferrite film $(d, e, f)$. In parts (a), (d) the curve 1 is with retardation, 2 is in quasistationary approximation. In parts (b), (c), (e), (f) the curve 1 is $\partial^{2} \omega / \partial k_{z}^{2}, 2$ is $\partial^{2} \omega / \partial k_{y}^{2}, 3$ is $\partial^{2} \omega / \partial k_{z} \partial k_{y}\left(\mathrm{~cm}^{2} / \mathrm{s}\right.$ units $)$.

The values of $k(\omega)$ calculated in the quasistationary approximation do not differ essentially from the exact ones, see Fig. 2, parts (a), (d). But the influence of the hybridization of the SDW with the EM waves leads mainly to the change of the values, especially, the sign, of the dispersion coefficient $\partial^{y} \omega / \partial k_{z}^{y}$.

Such a change of the sign occurs at the relatively high values of the wave numbers $k \approx 150 \mathrm{~cm}^{-1}$, as seen in Fig. 2, parts (b), (e). The transverse profiles of the wave change weakly. Moreover, the retarda- 
tion leads to an appearance of the cross-dispersion term $\partial^{2} \omega / \partial k_{z} \partial k_{y}$. Also the $y$-component of the group velocity occurs: $\partial \omega / \partial k_{y} \neq 0$ at $k_{y}=0$, but $\left|\partial \omega / \partial k_{y}\right| \ll\left|\partial \omega / \partial k_{z}\right|$. This occurs due to the asymmetry of the structure in the vertical direction.

Thus, to obtain the bright temporal soliton propagation and the realization of the wave collapse, it is necessary to choose the larger values of the wave numbers $k>100-150 \mathrm{~cm}^{-1}$.

\section{Numerical simulations}

The propagation of the SDW pulses transversely localized along $Y$-direction is under investigations. A possibility of the wave collapse of the SDW in the YIG films and in the hexaferrite ones has been checked by the simulations of the nonlinear parabolic equation, which corresponds to the expansion of $\omega=\omega\left(k_{z}, k_{y},|A|^{2}\right)$. In an undimensional form, this equation is

$$
\begin{aligned}
& \frac{\partial A}{\partial t}+v_{1} \frac{\partial A}{\partial z}+v_{2} \frac{\partial A}{\partial y}+\mathrm{i} g_{11} \frac{\partial^{2} A}{\partial z^{2}}+\mathrm{i} g_{22} \frac{\partial^{2} A}{\partial y^{2}} \\
& +\mathrm{i} g_{12} \frac{\partial^{2} A}{\partial z \partial y}+2 \mathrm{i}|A|^{2} A+\Gamma A=0 .
\end{aligned}
$$

Usually the transverse component of the group velocity is small: $\left|v_{2}\right| \ll\left|v_{1}\right|$, whereas the diffraction coefficient $g_{12}$ can be comparable with the dispersion one $g_{11}$ under the small values of the wave number $k \leq 100 \mathrm{~cm}^{-1}$. Equation (2) has been added by the boundary condition

$$
\begin{aligned}
& A(z=0, y, t)=A_{0} \exp \left(-\left(\left(t-t_{1}\right) / t_{0}\right)^{4}\right) \\
& \quad \times \exp \left(-\left(\left(y-y_{1}\right) / y_{0}\right)^{4}\right) .
\end{aligned}
$$

The numerical solution of (2) has been obtained with the splitting by the physical factors [12]:

$$
\begin{aligned}
& \frac{A^{p+1 / 3}-A^{p-1}}{2 \tau}+\hat{L}_{2} A^{p+1 / 3}+\hat{L}_{12} A^{p}=0 \\
& \frac{A^{p+2 / 3}-A^{p+1 / 3}}{2 \tau}+\mathrm{i} N|A|^{2} A^{p+2 / 3}+\Gamma A^{p+2 / 3}=0 \\
& \frac{A^{p+1}-A^{p+2 / 3}}{2 \tau}+\hat{L}_{3} A^{p+1}=0 \\
& \hat{L}_{1} \equiv v_{1} \frac{\partial}{\partial z}+\mathrm{i} g_{11} \frac{\partial^{2}}{\partial z^{2}}, \quad \hat{L}_{12} \equiv \mathrm{i} g_{12} \frac{\partial^{2}}{\partial z \partial y} \\
& \hat{L}_{3} \equiv v_{2} \frac{\partial}{\partial y}+\mathrm{i} g_{22} \frac{\partial^{2}}{\partial y^{2}} .
\end{aligned}
$$

Here $A^{p} \equiv A(p \tau), \tau$ is the temporal step. The stable 3-layer scheme has been used at the first fractional step, because of the term with the mixed derivatives $\partial^{2} A / \partial z \partial y$.

The results of the simulations are given in Figs. $3-5$ and in Figs. 7-9. The thickness of the film is $2 l=10 \mu \mathrm{m}$. The main obstacle for observing the soliton formation and the wave collapse is the dissipation of the SDW, which is low in the YIG films and quite high in the hexaferrite ones. One can see that for the SDW in the YIG films there

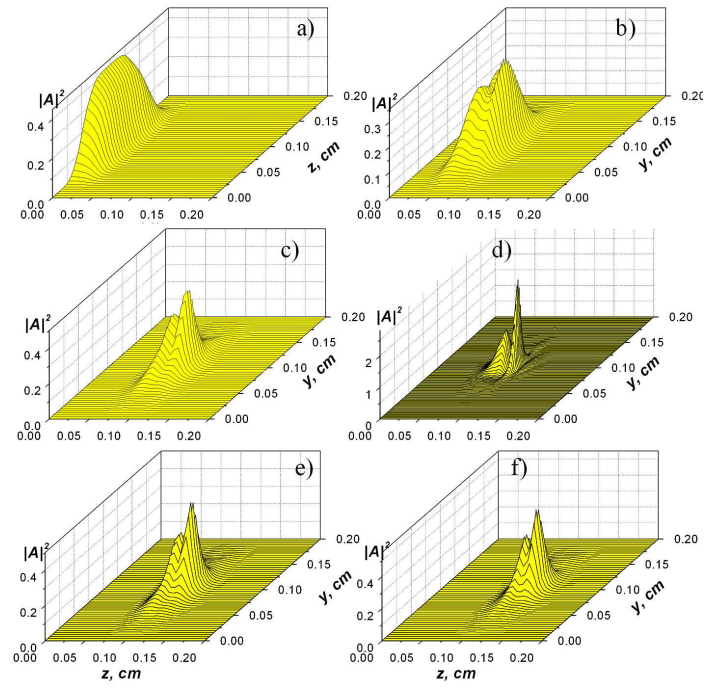

Fig. 3. Dynamics of the nonlinear SDW in the YIG film; $A_{0}=0.65$. Part (a) is at the time moment $t=$ $10 \mathrm{~ns}$, (b) $t=20 \mathrm{ns,} \mathrm{(c)} t=25 \mathrm{~ns}$, (e) $t=27 \mathrm{ns,} \mathrm{(f)} t=$ $29 \mathrm{~ns}$. Part (d) is for the focused pulse at $t=25 \mathrm{~ns}$.

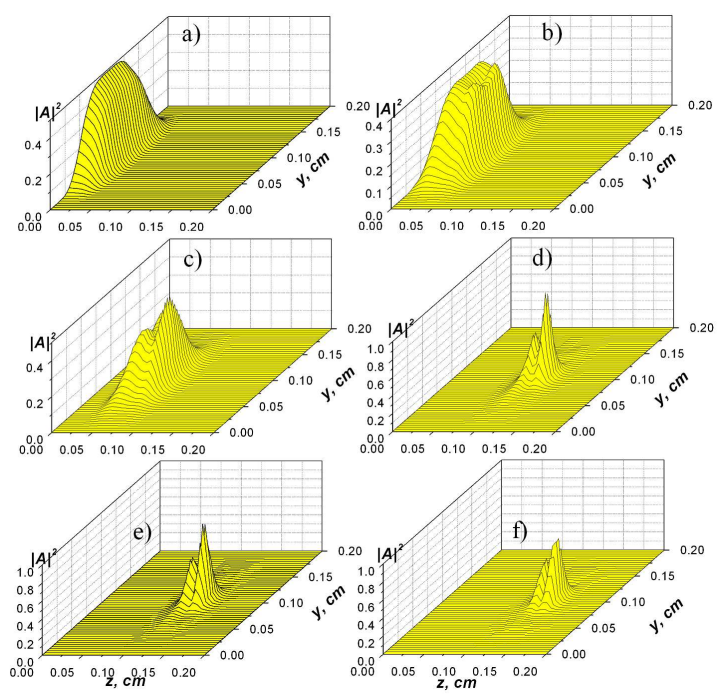

Fig. 4. Dynamics of the nonlinear SDW in the YIG film; $A_{0}=0.7$. Part (a) is at $t=10 \mathrm{~ns}$, (b) $t=15 \mathrm{ns,}$ (c) $t=20 \mathrm{~ns},(\mathrm{~d}) t=28 \mathrm{~ns},(\mathrm{e}) t=30 \mathrm{~ns}$, (f) $t=32 \mathrm{~ns}$.

exists the value of the input pulse amplitude $A_{0}$ when the pulse compression both in $Z$-direction and in $Y$-direction takes place, compare Fig. 3 and Figs. 4, 5. As a result, the nonlinearity ceases to be moderate, and the wave collapse occurs, see Fig. 5. In Figs. 3-5, the following values are used: the carrier frequency is $\omega_{0}=2.24 \times 10^{11} \mathrm{~s}^{-1}$, the central wave number is $k_{0}=300 \mathrm{~cm}^{-1}$, the dissipation of the YIG is $\delta=2 \times 10^{-4}, \omega_{M}=3.1 \times 10^{10} \mathrm{~s}^{-1}, \omega_{H}=$ $2 \times 10^{11} \mathrm{~s}^{-1} ; t_{0}=5 \mathrm{~ns}, y_{0}=0.075 \mathrm{~cm}$. The value $A=1$ corresponds to the ratio $\left(m_{z}-M_{0}\right) / M_{0}=-0.025$, where $m_{z}$ is $z$-component of the magnetization in the presence of SDW. 

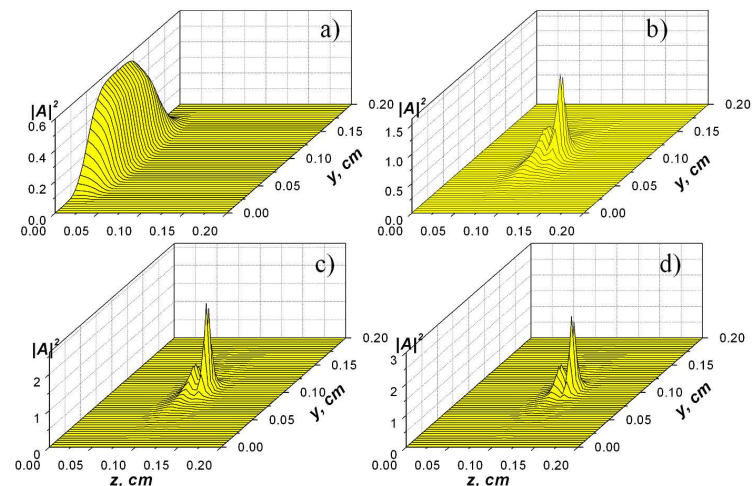

Fig. 5. Dynamics of the nonlinear SDW in the YIG film; $A_{0}=0.75$. Part (a) is at $t=10 \mathrm{ns,} \mathrm{(b)} t=25 \mathrm{ns,}$ (c) $t=27 \mathrm{~ns},(\mathrm{~d}) t=29 \mathrm{~ns}$.

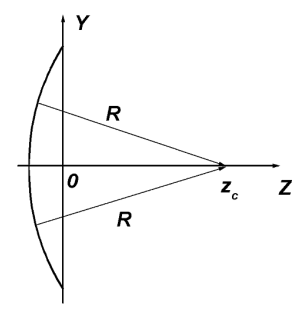

Fig. 6. Geometry of the focusing of the SDW. The parameter $R$ is the radius of antenna, $z_{\mathrm{c}}$ is the coordinate of the focus.

An efficiency of the self-action of the nonlinear pulses by means of additional focusing the pulses [13] is considered, too, see Fig. 6. When the exciting antenna for the SDW is circular it is possible to focus the beam of the SDW. The simplified approach is applied [13] to analyze this focusing, which is consistent with Eq. (2). Namely, the equivalent boundary condition at $z=0$ is used. The propagation difference for the rays at $z=0$ is written up to obtain this condition: $\Delta(y) \approx R-\left(z_{\mathrm{c}}^{2}+y^{2}\right)^{1 / 2}$. Here $R$ is the radius of the curvature of the antenna, $z_{\mathrm{c}}$ is the coordinate of the focus. This leads to the following phase difference: $\Psi(y) \approx-k_{\text {eff }} \Delta(y)$, where $k_{\text {eff }}$ is the effective longitudinal wave number that corresponds to the diffraction coefficient in Eq. (2). Thus, in the simplest model of the focusing, it is possible to use the additional mul-

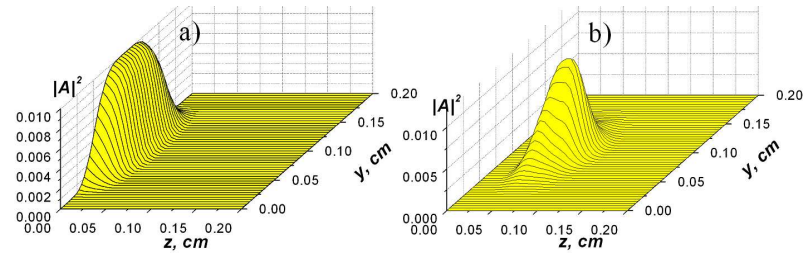

Fig. 7. The focusing of the linear pulse in the YIG film. Part (a) is at $t=10 \mathrm{~ns}$; part (b) is at $t=25 \mathrm{~ns}$, maximum compression.
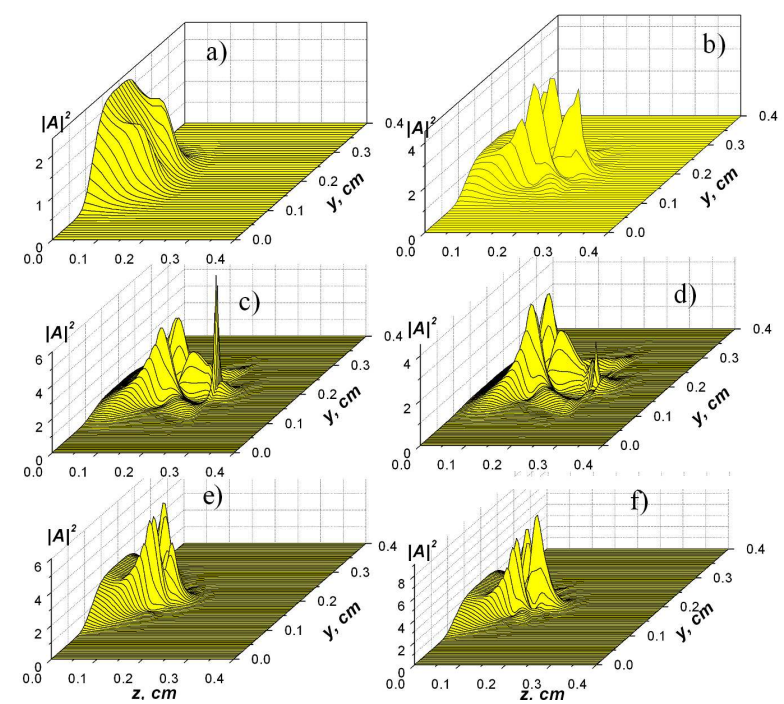

Fig. 8. Dynamics of the nonlinear SDW in the hexaferrite film; $A_{0}=1.5$. Part (a) is at $t=19.2 \mathrm{ns,}$ (b) $t=25.6 \mathrm{~ns}$, (c) $t=27.2 \mathrm{ns,}$ (d) $t=28.8 \mathrm{~ns}$. Parts (e), (f) are for the focused pulse at $t=20.8 \mathrm{~ns}$, $t=22.4 \mathrm{~ns}$.
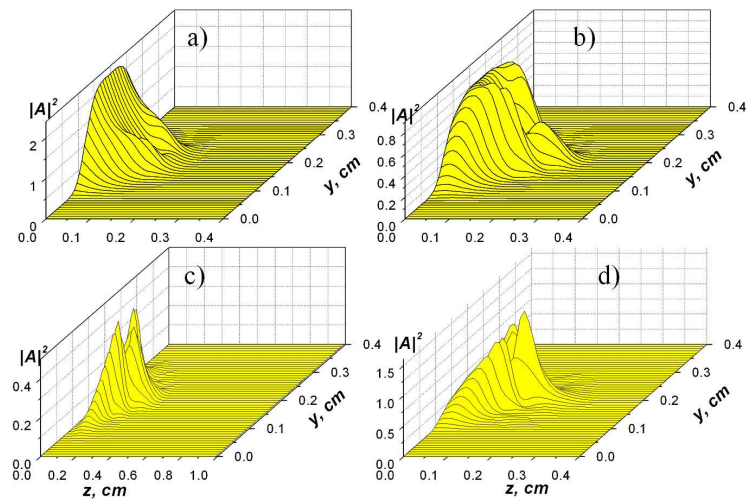

Fig. 9. Dynamics of the nonlinear SDW in the hexaferrite film; $A_{0}=1.5$. Higher dissipation. Part (a) is at $t=22.4 \mathrm{~ns}$, (b) $t=27.2 \mathrm{~ns}$, (c) $t=30.4 \mathrm{~ns}$. Part (d) is for the focused pulse at $t=28 \mathrm{~ns}$, the maximum compression.

tiplier in Eq. (3): $\exp (-\mathrm{i} k \Delta(y))$. In the undimensional form, the expression for $k_{\text {eff }}$ can be obtained from Eq. (2) as $k_{\text {eff }} \approx g_{22} /\left(2 v_{1}\right)$. As a result the multiplier takes the form $\exp \left(-\mathrm{i} g_{22} /\left(2 v_{1}\right) \Delta(y)\right)$.

In the case of the YIG films, the focusing leads to the essential increase of the nonlinear self-compression of the pulse. In Fig. 3, part (d), the distribution of $|A|^{2}$ is given for the time moment $t=25 \mathrm{~ns}$. The radius of the curvature of the input antenna is $R=0.25 \mathrm{~cm}$. One can see that by means of the initial focusing it is possible to reach the collapse at the lower values of the amplitude $A_{0}$, when comparing with the pulse without the initial phase modulation. In Fig. 7 the maximum compression is demonstrated for the modulated pulse of a 
small amplitude $A_{0}=0.1$. Only the weak increase of the amplitude of the linear pulse occurs there. This confirms the fact that in Fig. 3d namely the nonlinear pulse compression takes place.

In the hexaferrite films, the dispersion coefficients of the SDW are higher than for the YIG films. But the wave dissipation is one order higher, too, see Figs. 8, 9. Figure 9 corresponds to a more realistic value of the wave dissipation. The transverse compression of the SDW pulse occurs, i.e. the formation of the spatial solitons, but the wave collapse does not take place. In Figs. 8, 9 the values are: $\omega_{0}=2.1 \times 10^{11} \mathrm{~s}^{-1}, k_{0}=300 \mathrm{~cm}^{-1}$, the dissipation in the hexaferrite is $\delta=1 \times 10^{-3}$ (Fig. 8) and $\delta=2 \times 10^{-3}$ (Fig. 9), $\omega_{M}=8.8 \times 10^{10} \mathrm{~s}^{-1}, \omega_{H}=1.7 \times 10^{11} \mathrm{~s}^{-1}$; $t_{0}=5 \mathrm{~ns}, y_{0}=0.15 \mathrm{~cm}$. The value $A=1$ corresponds to $\left(m_{z}-M_{0}\right) / M_{0}=-0.01$ there.

The additional focusing can improve the compression of the nonlinear pulses in the hexaferrite films, see Fig. 8, parts (e), (f), and Fig. 9, part (d). The curvature radius of the antenna is $R=0.4 \mathrm{~cm}$ there. But the strong dissipation prevents the forming of the wave collapse even with the additional focusing. Nevertheless, this focusing could be useful in the devices based on the nonlinear pulses of the SDW, like [8].

When the exact wave dispersion was taken into account, the simulations have confirmed the results obtained within the parabolic approximation. Namely, the exact linear wave dispersion has been used as given in Fig. 2, parts (a), (d), instead of the parabolic approximation of Eq. (2). The dispersion of nonlinearity has been taken into account, too [14]. These simulations have been confirmed that the wave collapse can occur for the SDW in the hexaferrite films only at the dissipation $\delta \leq 5 \times 10^{-4}$.

\section{Conclusions}

The calculations of the dispersion and the diffraction coefficients of the SDW in the ferrite films have demonstrated that the bright soliton propagation and the wave collapse of the SDW in the millimeter wave range are possible only at the wave numbers $k>150 \mathrm{~cm}^{-1}$. The main obstacle for the realization of the wave collapse in the millimeter wave range is the wave dissipation. In the YIG films under quite high values of the bias magnetic fields the wave collapse of the nonlinear SDW is possible. In the hexaferrite films under the realistic parameters, the wave dissipation prevents the wave collapse; an occurrence of the spatial solitons only is possible there. The additional focusing of the input SDW pulses can lead to increasing nonlinear compression of the pulse.

\section{Acknowledgments}

This work was partially supported by SEP-CONACyT (Mexico). The authors are grateful to Acad. V.G. Bar'yakhtar for the interest to our work.

\section{References}

[1] Y.S. Kivshar, G.P. Agrawal, Optical Solitons, Acad. Press, New York 2003.

[2] K. Aoki, Nonlinear Dynamics and Chaos in Semiconductors, IoP Publ., Bristol 2001.

[3] Nonlinear Wave Phenomena and Chaos in Magnetic Materials, Ed. P.E. Wigen, World Sci., Singapore 1994.

[4] B.A. Kalinikos, N.G. Kovshikov, A.N. Slavin, J. Phys. Colloq. (France) 49, C8-1583 (1988).

[5] S.O. Demokritov, B. Hillebrands, A.N. Slavin, Phys. Rep. 348, 441 (2001).

[6] V. Grimalsky, Yu. Rapoport, A.N. Slavin, J. Phys. IV (France) 7, C1-393 (1997).

[7] O. Buettner, M. Bauer, S.O. Demokritov, B. Hillebrands, Yuri S. Kivshar, V. Grimalsky, Yu. Rapoport, A.N. Slavin, Phys. Rev. B 61, 11576 (2000).

[8] B.A. Kalinikos, A.N. Slavin, Appl. Phys. Lett. 79, 1576 (2001).

[9] A.A. Serga, S.O. Demokritov, B. Hillebrands, A.N. Slavin, Phys. Rev. Lett. 92, 117203 (2004).

[10] A.G. Gurevich, G.A. Melkov, Magnetization Oscillations and Waves, CRC Press, Boca Raton, CA 1996.

[11] V.G. Bar'yakhtar, B.A. Ivanov, Modern Magnetism. A Primer, Mir, Moscow 1985.

[12] G.I. Marchuk, Numerical Methods and Applications, CRC Press, Boca Raton, CA 1994.

[13] A.D. Boardman, Yu.G. Rapoport, V.V. Grimalsky, B.A. Ivanov, S.V. Koshevaya, L. Velasco, C.E. Zaspel, Phys. Rev. E 71, 026614 (2005).

[14] C.E. Zaspel, J.H. Mantha, Yu.G. Rapoport, V.V. Grimalsky, Phys. Rev. B 64, 064416 (2001). 